\title{
Speed of reaction diffusion in embryogenesis
}

\author{
Karen M. Page, ${ }^{1, *}$ Nicholas A. M. Monk, ${ }^{2, \dagger}$ and Philip K. Maini ${ }^{3,4, \ddagger}$ \\ ${ }^{1}$ Department of Mathematics, University College London, Gower Street, London WC1E 6BT, United Kingdom \\ ${ }^{2}$ Computational Systems Biology Group, The Kroto Research Institute, University of Sheffield, North Campus, Broad Lane, \\ Sheffield S3 7HQ, United Kingdom \\ ${ }^{3}$ Centre for Mathematical Biology, Mathematical Institute, 24-29 St. Giles', Oxford OX1 3LB \\ ${ }^{4}$ Oxford Centre for Integrative Systems Biology, Department of Biochemistry, South Parks Road, Oxford OX1 3QU, United Kingdom
}

(Received 14 August 2006; published 5 July 2007)

\begin{abstract}
Reaction diffusion systems have been proposed as mechanisms for patterning during many stages of embryonic development. While much attention has been focused on the study of the steady state patterns formed and the robustness of pattern selection, much less is known about the time scales required for pattern formation. Studies of gradient formation by the diffusion of a single morphogen from a localized source have shown that patterning can occur on realistic time scales over distances of a millimeter or less. Reaction diffusion has the potential to give rise to patterns on a faster time scale, since all points in the domain can act as sources of morphogen. However, the speed at which patterning can occur has hitherto not been explored in depth. In this paper, we investigate this issue in specific reaction diffusion models and address the question of whether patterning via reaction diffusion is fast enough to be applicable to morphogenesis.
\end{abstract}

DOI: 10.1103/PhysRevE.76.011902

PACS number(s): 87.18.La, 87.18.Hf, 87.15.Vv

\section{INTRODUCTION}

The vast majority of studies of reaction diffusion systems have been directed at analysis of their steady states. However, if these systems are to provide an explanation of biological patterning, they must also be able to produce patterns on a biologically realistic time scale. It is therefore important to consider the speed of formation of pattern [1].

A major limitation on the speed of the patterning process arises from the fact that most potential morphogens diffuse slowly in the embryo. Crick [2] addressed this issue in his classic 1970 paper, and showed that simple and facilitated diffusion of small molecules within and between cells are sufficient to account for the formation of stable concentration gradients around a localized source. Crick considered the time taken for a gradient in the concentration of a chemical species to be established through simple diffusion in a onedimensional domain with a source at one end and a sink at the other. Arguing from basic biophysical principles, Crick deduced that in a time of about three hours a gradient can be established over a distance of a millimeter or less, but not over a distance of a centimeter. More recently, Lander et al. [3] have obtained comparable results for the case of growth factors diffusing in the extracellular environment, but it remains unclear whether these results hold in a general reaction diffusion scenario. In a reaction diffusion system, every point in the spatial domain is a potential source of the chemical species concerned and so pattern formation does not depend directly on the movement of morphogen away from a localized source.

\footnotetext{
*Email address: kpage@math.ucl.ac.uk FAX: +44 2073871397.

†Email address: n.monk@sheffield.ac.uk

*Email address: maini@maths.ox.ac.uk
}

In many biological patterning scenarios, gradient establishment by simple diffusion is likely to be too slow (especially since many candidate morphogens are proteins such as growth factors that have relatively low diffusibility due to strong interactions with the extracellular matrix). As shown by Crick, the time taken to establish a steady state gradient on a spatial domain of length $L$ is proportional to $L^{2}$, and this imposes an upper bound on the size of domain over which pattern can be established by simple diffusion. Systems in which each point of the domain can potentially act as a source of morphogen can generate spatial gradients more quickly, since diffusion across the entire domain is not necessary. For example, such systems can generate traveling waves that propagate across the domain in a time proportional to $L$. While these traveling waves typically result in spatially uniform steady states, they can still generate stable gradients of cellular response if individual cells "integrate" the morphogen signal over time [4]. Such signal integration has recently been described in the graded response of cells to the growth factor Activin in Xenopus embryos [5].

In this paper we investigate the speed of pattern formation in two-component reaction diffusion systems, with the aim of exploring whether such mechanisms can generate stable patterns on time scales that are appropriate for embryonic development. In particular, we focus attention on the specific case of the initiation of the primitive streak in chick embryos [6]. We first use standard linear analysis to determine the speed of patterning, and then investigate the validity of the linear analysis estimates of the time scales and explore nonlinear pattern selection and growth. We establish a correlation between the wavelength of the pattern and the time taken for it to form, and deduce that it is not possible to form a long wavelength pattern in a short time. Finally, we look at a specific modification of the standard reaction diffusion model which can reduce the time taken to form a pattern of a given number of peaks. 


\section{LINEAR ANALYSIS AND NUMERICAL SIMULATION OF TWO-COMPONENT REACTION DIFFUSION SYSTEMS}

In this section we present results relating to the growth rates of pattern modes from perturbations around a homogeneous steady state. Using linear analysis, we show that the speed of pattern formation depends on the wave number of the pattern and on the spatial discretization. We focus our attention on a two-component reaction diffusion system in one spatial dimension with zero flux boundary conditions. Following nondimensionalization of the system in space, such that the spatial domain becomes $[0,1]$, the general system is represented by the following equations:

$$
\begin{gathered}
u_{t}=f(u, v)+D_{u} u_{x x}, \\
v_{t}=g(u, v)+D_{v} v_{x x}, \\
u_{x}=v_{x}=0 \text { at } x=0,1,
\end{gathered}
$$

where $u$ and $v$ are the concentrations of the two morphogens, $D_{u}$ and $D_{v}$ are the corresponding diffusion coefficients, and $f(u, v)$ and $g(u, v)$ encode the reaction kinetics between $u$ and $v$.

We study three different kinetic schemes. Our primary example, which we study throughout the paper, uses GiererMeinhardt (GM) kinetics. [7] The GM model has pure activator-inhibitor kinetics (that is, the activator upregulates both the activator and inhibitor, while the inhibitor downregulates both). We also study two example models with cross kinetics (where the activator upregulates itself, but downregulates the inhibitor and the inhibitor likewise upregulates the activator and downregulates itself). These are the Schnakenberg or depletion [8,7] and glycolysis [9] models. Following nondimensionalization (see Appendix A for details) the forms of $f$ and $g$ for these models are as follows:

(i) Gierer-Meinhardt,

$$
f(u, v)=r\left(\frac{u^{2}}{v}+1\right)-\mu u, \quad g(u, v)=r u^{2}-\nu v
$$

(ii) Schnakenberg or delpletion,

$$
f(u, v)=r\left(u^{2} v+1\right)-\mu u, \quad g(u, v)=\delta-r u^{2} v
$$

(iii) glycolysis,

$$
f(u, v)=r u^{2} v+\nu v-\mu u, \quad g(u, v)=r\left(1-u^{2} v\right)-\nu v .
$$

In each case, the parameters $r, \mu, \nu$, and $\delta$ are positive.

When performing numerical simulations of these equations, we used the numerical algorithms group (NAG) library routine D03PCF. This routine uses finite differences for spatial discretization and employs the method of lines to reduce the system of PDEs to a system of ODEs. The ODE system is solved using a backward differentiation formula method. An error tolerance of $10^{-7}$ was specified and the accuracy of the numerics was verified by performing each simulation with an error tolerance of $10^{-9}$ instead.

\section{A. Spatially homogeneous steady states and Jacobian matrices}

In order to calculate linear growth rates of periodic patterns in these systems, we first find the spatially homogeneous steady states and Jacobian matrices [10]. For each model, the spatially homogenous steady states $\left(u_{0}, v_{0}\right)$ and corresponding Jacobian matrices evaluated at $\left(u_{0}, v_{0}\right)$ are as follows:

(i) Gierer-Meinhardt,

$$
\begin{gathered}
u_{0}=\frac{r+\nu}{\mu}, \quad v_{0}=\frac{r(r+\nu)^{2}}{\mu^{2} \nu}, \\
J=\left(\begin{array}{cc}
\mu \frac{\nu-r}{\nu+r} & -\frac{\nu^{2}}{r}\left(\frac{\mu}{\nu+r}\right)^{2} \\
2 r \frac{r+\nu}{\mu} & -\nu
\end{array}\right) ;
\end{gathered}
$$

(ii) Schnakenberg,

$$
\begin{gathered}
u_{0}=\frac{r+\delta}{\mu}, \quad v_{0}=\frac{\delta \mu^{2}}{r(\delta+r)^{2}}, \\
J=\left(\begin{array}{cc}
\mu \frac{\delta-r}{\delta+r} & r \frac{(\delta+r)^{2}}{\mu^{2}} \\
-2 \frac{\delta \mu}{\delta+r} & -r \frac{(\delta+r)^{2}}{\mu^{2}}
\end{array}\right) ;
\end{gathered}
$$

(iii) Glycolysis,

$$
\begin{gathered}
u_{0}=\frac{r}{\mu}, \quad v_{0}=\frac{r \mu^{2}}{r^{3}+\nu \mu^{2}}, \\
J=\left(\begin{array}{cc}
\mu \frac{r^{3}-\nu \mu^{2}}{r^{3}+\nu \mu^{2}} & \nu+\frac{r^{3}}{\mu^{2}} \\
-2 \frac{r^{3} \mu}{r^{3}+\nu \mu^{2}} & -\nu-\frac{r^{3}}{\mu^{2}}
\end{array}\right) .
\end{gathered}
$$

\section{B. Bounds on pattern growth}

In this section, we present bounds on the linear growth rate of spatially periodic patterns (modes) about the homogeneous steady state. We first find bounds on the linear growth rate of all modes, both in general and for each specific model described in the previous section. We then consider the effects on pattern growth of the initial perturbations (noise) about the homogeneous steady state and of the spatial discretization used in simulations of the models. Details of the analysis are given in Appendix B.

\section{Linear growth rate of any mode}

The linear growth rate of any growing mode in a twocomponent reaction diffusion system is bounded above by the rate of growth in the corresponding system in the limit as activator diffusion tends to zero. This limiting growth rate is given by the first $\left(J_{11}\right)$ entry in the Jacobian matrix of the 
linearized system. The maximum growth rate $\lambda_{\max }$ (over all modes, without considering the restrictions placed by the boundary conditions) is given by

$$
\lambda_{\max }=f_{u}+\frac{D_{u}\left(f_{u}-g_{v}\right)-2 \sqrt{-f_{v} g_{u} D_{u} D_{v}}}{D_{v}-D_{u}} \leqslant f_{u} .
$$

The inequality must hold in order for the parameters to be in the Turing space. For all three of the example models described in the previous section this implies that $\lambda_{\max } \leqslant \mu$.

This bound is on the growth rate of the fastest growing mode. However, this mode has wave number $k_{*}$ where

$$
\begin{aligned}
k_{*}^{2} & =\frac{g_{v}-f_{u}+\left(D_{v}+D_{u}\right) \sqrt{-f_{v} g_{u} / D_{u} D_{v}}}{D_{v}-D_{u}} \\
& \sim \sqrt{\frac{-f_{v} g_{u}}{D_{v}}} \frac{1}{\sqrt{D_{u}}} \text { as } D_{u} \rightarrow 0 .
\end{aligned}
$$

It therefore leads to a many-peaked pattern as $D_{u}$ gets small (i.e., as we get near to attaining the limiting growth rate).

In Fig. 5, we give examples of $k^{*} / \pi$, the linear analysis estimate of the number of half peaks, and $\lambda_{\max }$, the estimated linear growth rate of the pattern, and compare these estimates to the results of numerical simulations.

In many important biological scenarios (for example, primitive streak initiation in the chick [6]) the system must initiate a pattern that comprises either a single peak or a small number of peaks. To account for such patterning, we require a system that can generate a pattern of only a few peaks in a biologically realistic time. Making the assumption that the growth of a pattern of given period will be limited by the growth rate of the linear mode of the same period (we evaluate the validity of this assumption later), we consider bounds on the linear growth rates of given modes. In this case, we can derive bounds depending crucially on the diffusion coefficients. This is important since those chemical species which are considered candidate morphogens in the early embryo are expected to have small diffusion coefficients, whereas little is known about the sizes of the kinetic parameters.

\section{Mode-specific linear growth rates}

For the Gierer-Meinhardt model [7], the linear growth rate of the mode with wave number $k$ is restricted (see Appendix B) by

$$
\lambda(k) \leqslant \frac{D_{v}(\sqrt{2}-1) k^{2}}{2} .
$$

On a domain of length $L$ with zero flux boundary conditions, $k$ will take values $n \pi / L, n=1,2 \ldots$ On a periodic domain of length $L$ it will take only values which are multiples of $2 \pi / L$. Equation (13) clearly places a much more stringent bound on the growth of few-peaked modes than the general bound of Eq. (11).

If we look, for instance, at the rate of establishment of a gradient pattern (i.e., a single-peaked pattern) on an effectively one-dimensional domain of length $L$ with zero flux boundary conditions, we find, substituting $k=\pi / L$ into Eq.
(13), that $\lambda \leqslant 2 D_{v} / L^{2}$. Standard linear analysis tells us that the initial growth of the $n \pi$ mode about the uniform steady state is described by $A_{n}(t)=A_{n}(0) e^{\lambda(n \pi) t}$, where $A_{n}(t)$ is the amplitude of the $n \pi$ mode at time $t$. Thus the time taken for the $n \pi$ mode to reach $y \%$ of the uniform steady state value is given by $\frac{1}{\lambda} \ln \left[\frac{u_{0} y}{100 A_{n}}\right]$. The time taken for a gradient to be established clearly depends on the initial amplitude of the gradient mode, although $1 / \lambda$ gives an indication of the time scale of establishment.

\section{Dependence of linear growth rates on initial conditions}

In de novo patterning, the gradient mode is present in the initial conditions due to noise in the system. The initial amplitude of each mode depends on the nature of the initial perturbations to the homogeneous steady state, and it is difficult to assess the exact nature of this noise experimentally. Therefore unless the statistics of the Fourier components of the initial conditions are insensitive to the type of noise, it will be difficult to assess the initial Fourier components and hence the time taken for a pattern to form. If the noise is randomly distributed at each cell (discretization point or set of such points) in the domain and is white with mean 0 and expected magnitude $z \%$ of the uniform steady state value, then we find that the gradient mode initially has an amplitude of approximately $z \sqrt{2 / m} \%$ of the uniform steady state value, where $m$ is the number of cells in the domain (see Appendix C).

This result demonstrates that for noisy initial conditions of this type, the initial Fourier components and the time taken to form a pattern are sensitive to the number of cells in the domain. If we consider the cellular and stochastic nature of the system to be important, then it is also important to consider the effects that these features have on the equations as well as on the initial conditions. Turing showed that for a reaction diffusion system, the contribution of an additive noise term in the Fourier components was significant only in a small initial time window [11]. Thereafter, the growing modes to which the noise contributes initially become sufficiently large that any newly added noise component is insignificant. Thus we hypothesize that partial differential equation (PDE) models may provide a fairly accurate description of noise-driven de novo patterning only after an initial "waiting time," during which the system should be studied using stochastic methods. In general, the final (steady state) patterns formed by reaction diffusion systems are insensitive to the details of noise present in the system (at least for low wave-number patterns and reasonably white noise) and so it is typically assumed that it is possible to ignore the initial stochastic phase in analyzing those patterns. However, in order to calculate the time taken for patterns to form, we require a waiting time and PDE initial conditions to be calculated using stochastic analysis.

In the following, we shall assume that the main effect of the stochasticity is to give rise to small amplitude Fourier components in the initial conditions. Furthermore, we shall assume that the cellular nature of the system has little effect on the time taken for a pattern to be produced, except through its effect on the spatial scale of fluctuations in the initial conditions. Thus we will suppose that the initial am- 
plitude of a Fourier component is approximately $z \sqrt{2 / m} \%$, as suggested by the analysis in Appendix C.

We assume that cells can interpret a gradient when it has amplitude $y \%$ of the uniform steady state value. The amplitude of the gradient mode will thus have to increase about $\sqrt{\frac{m}{2}} \frac{y}{z}$-fold in order for an interpretable gradient to appear. This means that $\lambda t$ (where $t$ is the time) must reach a value of $\ln \left(\sqrt{\frac{m}{2}} \frac{y}{z}\right)=\ln \left(\frac{y}{z}\right)+\frac{1}{2} \ln \left(\frac{m}{2}\right)$, assuming linear growth.

This dependence on the number of cells in the domain means that care must be taken not only in modeling biological cellular systems using continuum equations, but also in the numerical solution of continuum equations using discretization. It is common practice to generate noise in the initial conditions by introducing a random variable at each spatial discretization point. It should be noted that this will lead to different times taken for pattern formation as the number of discretization points is varied and is different from generating random fluctuations in the magnitude of the Fourier components.

Since we only have a logarithmic dependence on $m$ in the time taken for a pattern to form, we only need to know its size to an order of magnitude. We will fix $m$ in the following analysis at 100 , corresponding to a domain length of approximately $3 \mathrm{~mm}$ (assuming a cell diameter of $30 \mu \mathrm{m}$ ) and assume that this will give a reasonable approximation for domains in the range of $1 \mathrm{~mm}$ to $1 \mathrm{~cm}$. Thus $\lambda t$ must reach a value of $\ln (y / z)+\ln (50) / 2$. From our previous bound on $\lambda$ we deduce that $t \geqslant A L^{2} / D_{v}$, where $A$ is a dimensionless constant whose value is about $0.5[\ln (y / z)+\ln (50) / 2]$. If we assume that $y$ is likely to be larger than $z$, we see that $A$ $>0.97$. For simple diffusion on a one-dimensional domain with a source at one end and a sink at the other, Crick obtained patterning times of the same form, with values of $A$ in the range of approximately $0.1-0.5$ [3]. Our analysis shows that the Gierer-Meinhardt model generates a gradient on an even slower time scale, although it does so de novo, being triggered simply by noise, rather than an initial polarity of the system.

\section{Mode-specific linear growth rates for general models}

The bound given in inequality (13) applies only for the Gierer-Meinhardt model. We can obtain a (albeit weaker) bound for general reaction diffusion models:

$$
\lambda(k)<\frac{D_{v} k^{2}}{\beta-1},
$$

where $\beta=\frac{\left|g_{u} f_{v}\right|}{f_{u}^{2}} \quad$ For the three example models (Gierer-Meinhardt, Schnakenberg or depletion, and glycolysis), the conditions for diffusion-driven instability imply that $\beta>2$ and hence from Eq. (14) we obtain

$$
\lambda(k)<D_{v} k^{2} .
$$

Figure 1 shows an example plot of the dispersion relation $(\lambda$ vs $k$ ) for the Gierer-Meinhardt model, along with the bounds given in Eqs. (11), (13), and (15). In the context of embryonic patterning, the latter two bounds are of greater interest, since the growth rates are restricted by the inhibitor

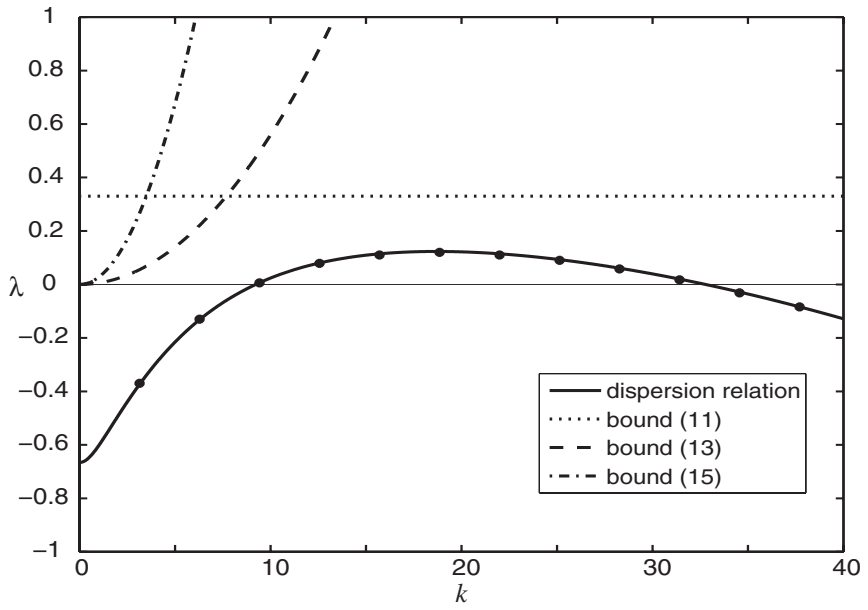

FIG. 1. Dispersion relation of the Gierer-Meinhardt model equations with parameters $\nu=2.0, \mu=0.33, r=0.001, d=0.01$, and $D_{v}$ $=0.027$ along with the linear analysis bounds on the growth rates given in Eqs. (11), (13), and (15). The parameter values were chosen such that the $6 \pi$ mode had the largest growth rate and the inhibitor diffusion coefficient was as large as considered biologically plausible.

diffusion rate. It is likely that the diffusion rates are restricted to be less than $10^{-6} \mathrm{~cm}^{2} / \mathrm{s}$, whereas we have little idea of the size of the kinetic parameters. We see from the figure that all of the bounds are quite weak, but that Eq. (13) gives the best bound on the growth rates of the lower modes.

Inequality (15) means that the establishment of a gradient on a domain of length $L$ with zero flux boundary conditions will take a time $t>A L^{2} / D_{v}$ where $A$ is approximately $[\ln (y / z)+\ln (50) / 2] / \pi^{2}$, which for $y>z$ is at least 0.2. However, this general bound is not very stringent. The inequality (14) gives the same value $A>0.2$ for the Gierer-Meinhardt model. This is clearly much less stringent than the heuristic bound obtained in the previous section. We show in Fig. 2 an example of how the bounds compare to the actual times taken for the gradient to reach $10 \%$ amplitude in simulations of the Gierer-Meinhardt model system. In the simulations, we used initial conditions with random fluctuation of $\pm 10 \%$ amplitude around the homogeneous steady state value at each discretization point. Five simulations with each of $m$ $=31,101$, and 1001 discretization points were performed. These results show that for the parameter set used, the actual times taken for the pattern to reach $10 \%$ amplitude are much greater than the bounds that we have obtained.

Even though the general bound is not stringent, if we take a generous upper bound on the inhibitor diffusion coefficient of $10^{-6} \mathrm{~cm}^{2} / \mathrm{s}$, then, on a domain of length $3 \mathrm{~mm}$, we get $t$ $>1.8 \times 10^{4} \mathrm{~s}$, which is about five hours. However, the bounds are weak and unlikely to be attainable. Certainly, the reaction diffusion systems that we have studied appear to pattern on a significantly longer time scale than this. However, our bounds suggest that reaction diffusion could be a plausible mechanism for patterning on smaller length scales. For inhibitor diffusion coefficients of the order of $10^{-8} \mathrm{~cm}^{2} / \mathrm{s}$ or higher, patterning could be attained in a plausible time on domains of about $100 \mu \mathrm{m}$ (for a potential example, see [12]). 


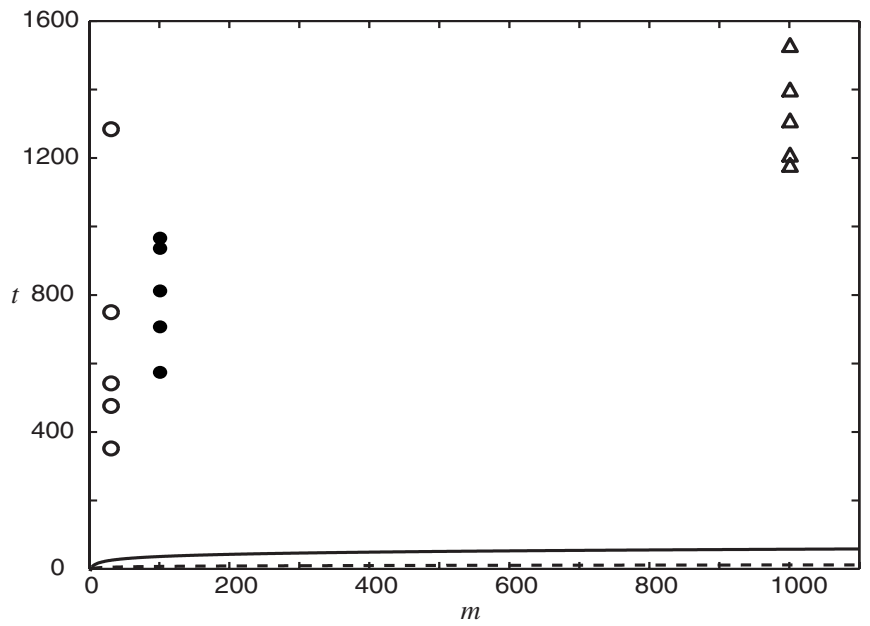

FIG. 2. Dependence of the time taken for the amplitude of the gradient mode to reach $10 \%$ of the uniform steady state value in the activator on the number of spatial discretization points $m$ in simulations of the Gierer-Meinhardt model equations. Parameters used: $\nu=0.05, \mu=0.01, r=0.001, d=0.01$, and $D_{v}=0.027$, and $m=31$ (open circles), $m=101$ (filled circles), and $m=1001$ (triangles). Also shown are the linear analysis bounds $t>\frac{1}{2}\left[\ln \left(\frac{y}{z}\right)+\frac{1}{2} \ln \left(\frac{m}{2}\right) \frac{L^{2}}{D_{v}}\right]$ (solid line), which is specific to the Gierer-Meinhardt model and $t$ $>\frac{1}{\pi^{2}}\left[\ln \left(\frac{y}{z}\right)+\frac{1}{2} \ln \left(\frac{m}{2}\right) \frac{L^{2}}{D_{v}}\right]$ (dashed line), which applies to the other example models, but is weaker.

\section{Influence of initial conditions and noise in the system}

As mentioned in the previous section, the initial conditions influence the time taken for pattern to develop. Specifically, it is often the case that the growing pattern is dominated by the fastest (exponentially) growing mode (see below). In this case, the time taken for the pattern to reach a certain threshold amplitude (capable of interpretation by the cells) depends crucially on the initial amplitude of that mode. In order to calculate the patterning times then, we need to be able to calculate the size of the Fourier components of the initial data.

So far, we have assumed that the initial conditions involve random perturbations about the uniform steady state. In our numerical simulations, we implement this by considering the noise in each variable to be an independent random variable uniformly distributed between $\pm z \%$ of its uniform steady state value at each point in our discretization. In the following analysis, we consider noise which is added pointwise at each cell or discretization point of the system at a level proportional to the uniform steady state value. For each species, we have

$$
u(r)=[1+\xi(r)] u_{0}, \quad r=1, \ldots, p,
$$

where $u$ is the concentration of the species, now considered as a function of the cell or discretization node number $r, u_{0}$ is the uniform steady state value, $p$ is the number of cells or nodes, and $\xi$ is a random variable such that the $\xi(r)$ are independent with zero mean.

Clearly, each time we run our simulation (or look at a different individual biological system), the initial data will be different. We cannot therefore exactly determine a priori the
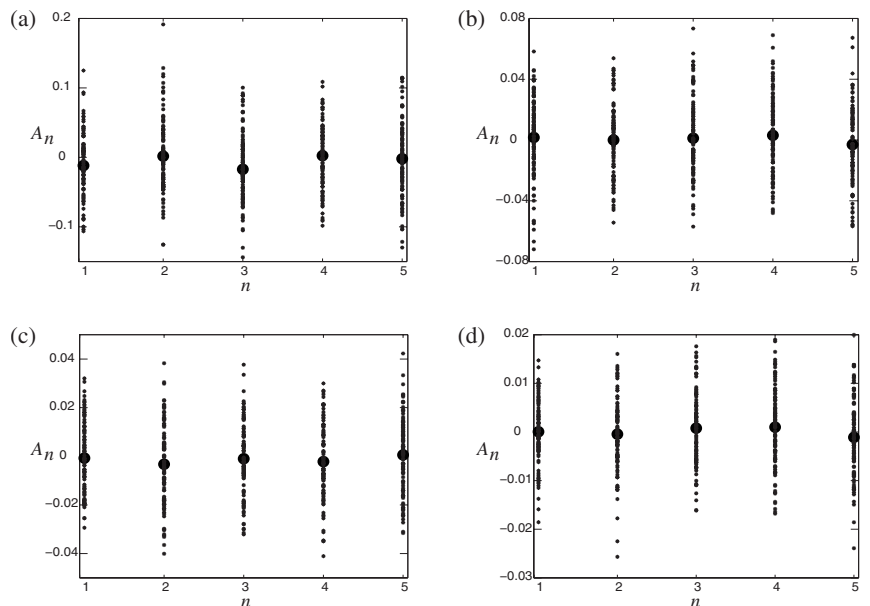

(e)

\begin{tabular}{|c|c|c|c|c|c||c|}
\hline$p \backslash n$ & $\mathbf{1}$ & $\mathbf{2}$ & $\mathbf{3}$ & $\mathbf{4}$ & $\mathbf{5}$ & $\mathbf{A n}$ \\
\hline $\mathbf{1 0 1}$ & $2.0 \times 10^{-3}$ & $2.9 \times 10^{-3}$ & $2.5 \times 10^{-3}$ & $2.2 \times 10^{-3}$ & $3.0 \times 10^{-3}$ & $2.4 \times 10^{-3}$ \\
\hline $\mathbf{4 0 1}$ & $6.9 \times 10^{-4}$ & $5.8 \times 10^{-4}$ & $6.7 \times 10^{-4}$ & $6.6 \times 10^{-4}$ & $6.5 \times 10^{-4}$ & $5.9 \times 10^{-4}$ \\
\hline $\mathbf{1 0 0 1}$ & $2.1 \times 10^{-4}$ & $2.7 \times 10^{-4}$ & $2.3 \times 10^{-4}$ & $2.4 \times 10^{-4}$ & $2.4 \times 10^{-4}$ & $2.4 \times 10^{-4}$ \\
\hline $\mathbf{4 0 0 1}$ & $4.1 \times 10^{-5}$ & $5.8 \times 10^{-5}$ & $5.9 \times 10^{-4}$ & $6.8 \times 10^{-5}$ & $6.1 \times 10^{-5}$ & $5.9 \times 10^{-5}$ \\
\hline
\end{tabular}

FIG. 3. Scatter plots of the amplitudes of the Fourier components $A_{1}$ to $A_{5}$ for initial data that are randomly distributed at each of $p$ discretization points about $u_{0}=6.0$, with uniform distribution between $\pm 10 \%$ of $u_{0}$. (a) $p=101$, (b) $p=401$, (c) $p=1001$, and (d) $p=4001$. For each set of amplitudes, the sample mean is shown by the large filled circle. (e) Table of the sample variance for each Fourier component $A_{n}$ as a function of $p$, together with the analytical estimate of the variance (final column: An).

magnitude of a given Fourier mode in a specific set of initial data, but instead we attempt to determine the statistics of the mode amplitude. The details of this analysis are presented in Appendix C.

We find that as we increase the number of cells in our system or spatial points in our discretization, the Fourier components become vanishingly small with probability 1 . This means that the time taken to form a pattern will increase unboundedly with $p$. While real biological systems have only a finite number of cells and hence patterning will occur in finite time, it is nonetheless clear that this time will depend on the details of the noise present in the system.

We note that if the noise at each cell of the system is Gaussian white noise (i.e., it is normally distributed), then, since the sum of independent normal random variables is a normal random variable, the Fourier components will also have Gaussian distributions. Since we know the mean and variance of the Fourier components [to $O(1 / p)$ ], we know completely their distributions. We can, for example, work out the expected magnitude of a Fourier component:

$$
\begin{aligned}
E\left(\left|A_{n}\right|\right) & =\frac{1}{\sigma\left(A_{n}\right) \sqrt{2 \pi}} \int_{-\infty}^{\infty}|x| e^{-x^{2} / 2 \sigma^{2}\left(A_{n}\right)} d x+O\left(\frac{1}{p}\right) \\
& =\sqrt{\frac{2}{\pi}} \sigma\left(A_{n}\right)+O\left(\frac{1}{p}\right)=\frac{2 u_{0} \sigma(\xi)}{\sqrt{p \pi}}+O\left(\frac{1}{p}\right) .
\end{aligned}
$$

Figure 3 shows a sample set of the Fourier components $A_{1}-A_{5}$, obtained from numerically generated initial data with 


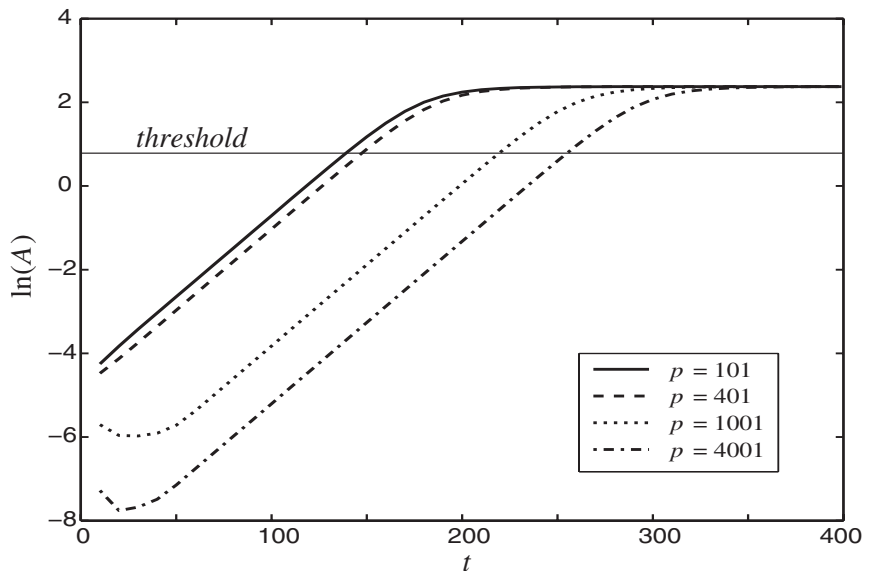

FIG. 4. Dependence of the growth rate of patterns on the number of spatial discretization points used in simulations. The plot shows the growth of the natural logarithm of the amplitude $A$ of the pattern over time in simulations of the Gierer-Meinhardt model with zero flux boundary conditions and parameters $\nu=0.05, \mu=0.01$, $D_{v}=0.027, d \equiv D_{u} / D_{v}=0.01$, and $r=0.001$. The initial conditions are random noise about the uniform steady state generated at each discretization point and uniformly distributed between $\pm 1 \%$ of the steady state value. The number of discretization points $p$ takes the values $101,401,1001$, and 4001 . The plot shows that there is a marked decrease in the growth rate of pattern as the number of discretization points increases. For illustration, an arbitrary threshold at which cells might respond to the activator is shown. In each case, the final pattern developed was of the form of mode 1 (a gradient).

(a) $p=101$, (b) $p=401$, (c) $p=1001$, and (d) $p=4001$. In each case, the sample mean and variance have been calculated; the distributions for each component are very similar, their means are close to zero and their variances show good agreement with our analytical estimate of $\frac{2 u_{0}^{2}}{p} \operatorname{Var}(\xi) . \xi$ is uniformly distributed on $[-0.1,0.1]$ and so has variance $=3.3$ $\times 10^{-3}$ and $u_{0}=6.0$.

Figure 4 shows the logarithmic amplitude of the activator against time in simulations with initial data generated on a grid of $p$ points for $p=101,401,1001$, and 4001. These results show clearly that as the number of discretization points (or biological cells) increases, there is a marked increase in the time taken for the activator concentration to reach an arbitrary threshold.

\section{HOW WELL DOES LINEAR ANALYSIS PREDICT THE GROWTH RATE OF PATTERNS?}

In this section we simulate the model equations numerically to see whether the patterns formed correspond to those predicted by linear analysis and we calculate the rates of growth of the patterns and compare them with the predictions from linear analysis. We use a discretization of 1001 points and the initial conditions are given by random perturbations about the uniform steady state values of both activator and inhibitor. The random perturbations are independent at each point of the discretization and are uniformly distributed between $\pm 5 \%$ of the uniform steady state values. We did not vary the number of spatial points, since this would alter the initial conditions and give rise to slightly different pattern amplitude profiles.

We consider the Gierer-Meinhardt model equations (1) and (2) on a one-dimensional spatial domain with zero flux boundary conditions and we nondimensionalize in space, such that the domain becomes $[0,1]$, and in time with a fixed time scale of $10^{4} \mathrm{~s}$ (just under three hours). This time scale is arbitrary, but is chosen to match the typical time scale of patterning in many embryonic patterning processes. We have chosen a (nondimensional) value of 0.027 for $D_{u}$, since this corresponds to an inhibitor diffusion coefficient of $10^{-6} \mathrm{~cm}^{2} / \mathrm{s}$ on a domain of length $6 \mathrm{~mm}$ (the approximate circumference of the marginal zone of the chick blastodisc before primitive streak initiation [6]). We fix the ratio of diffusion coefficients $d \equiv D_{u} / D_{v}=0.01$ and the nonlinear activator and inhibitor production parameter $r=0.001$. We vary the decay rates $\mu$ and $\nu$, so that linear analysis predicts selection of modes 1 to 6 .

Figure 5 displays the results of two example simulations (further simulations can be found in [13]), showing the steady state activator profile [Figs. 5(a) and 5(c)] and a plot of $\ln \left(u_{\max }-u_{\min }\right)$ against time [Figs. 5(b) and 5(d)], where $u_{\max }$ and $u_{\min }$ are the maximal and minimal values of the activator, respectively. The gradient gives a measure of the rate of growth: in a linear system, the growth would be exponential and our logarithm plot would hence be a straight line with gradient equal to the growth rate. According to the linear theory,

$$
u(x, t)=\sum_{n} A_{n} e^{\lambda_{n} t} \cos (n \pi x) \approx A_{n_{*}} e^{\lambda_{\max } t} \cos \left(n_{*} \pi x\right),
$$

where $A_{n}$ is the initial amplitude of the $n$th Fourier mode, $\lambda_{n}$ is its growth rate, and $n_{*}$ corresponds to the mode with maximal growth rate $\lambda_{\max }$. Thus

$$
u_{\max }-u_{\min } \approx 2 A_{n_{*}} e^{\lambda_{\max } t}
$$

and

$$
\ln \left(u_{\max }-u_{\min }\right)=\ln \left(2 A_{n_{*}}\right)+\lambda_{\max } t .
$$

In each simulation, the activator undergoes a period of exponential growth. The growth rates during this phase, calculated from the numerical data, are given in the caption of Fig. 5. Figures 5(e) and 5(f) show the growth rates of individual modes, as predicted by linear analysis for the given parameters. The linear analysis estimates of $\lambda_{\max }$ and $n_{*}$ are $\lambda_{\text {max }}=2.8 \times 10^{-3}$ and $n_{*}=0.92$ (parameter set 1) and $\lambda_{\max }$ $=0.12$ and $n_{*}=5.9$ (parameter set 2). We see that the linear analysis estimate for the number of peaks is a good predictor of the final pattern (note that the estimates take no account of the boundary conditions and merely give the location of the maximum of the dispersion relation, hence the nonintegral values of $n$ ).

Figure 6 shows the growth of modes 4-8 for the parameters used in Fig. 5(c) and illustrates how the mode with fastest linear growth rate is selected. In our simulation with $\nu=0.8, \mu=0.13$ (see [13]), the fastest-growing mode is not 
(a)

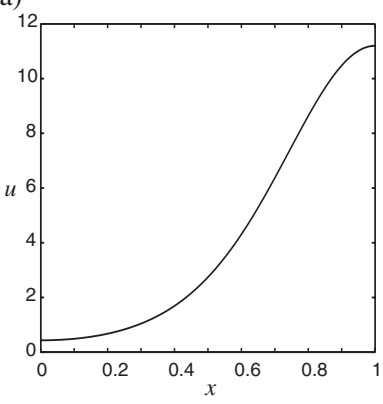

(c)

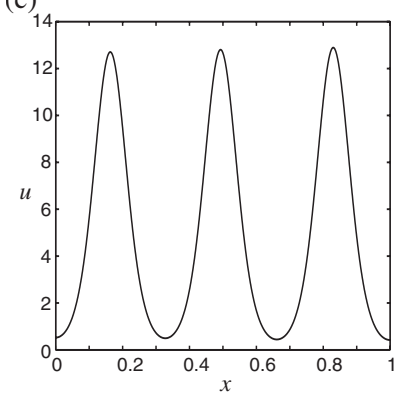

(e)

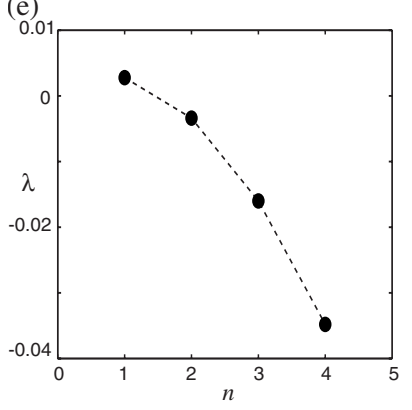

(b)

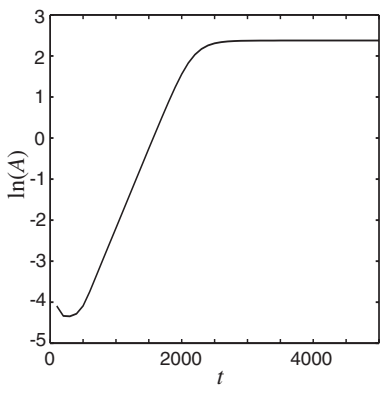

(d)

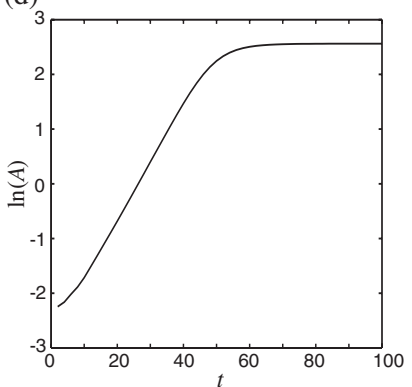

(f) 0.2

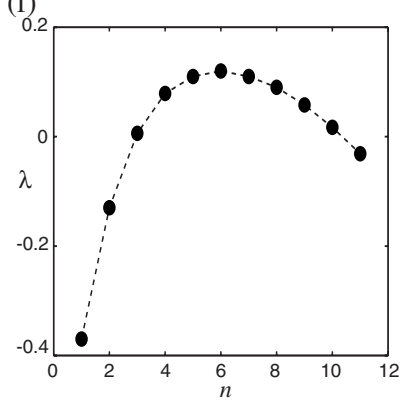

FIG. 5. (a)-(d) Steady state activator profiles [(a) and (c)] and growth of the natural logarithm of pattern amplitude [(b) and (d)] for the Gierer-Meinhardt model (1) and (2). (e), (f) Linear analysis estimates of growth rates of each mode $n$. Parameter set 1: $\nu$ $=0.05, \mu=0.01$ [plots (a), (c), and (e)]. Gradient of linear portion of (b) $=3.8 \times 10^{-3}$. Parameter set 2: $\nu=1.5, \mu=0.25$ [plots (b), (d), and (f)]. Gradient of linear portion of $(\mathrm{d})=8.24 \times 10^{-2}$.

selected, however the mode which is selected has a growth rate only very slightly less than that of the fastest-growing mode. With different (random) initial conditions the fastestgrowing mode is selected (see [13]).

We can see in Fig. 6 that, in the initial linear phase, the gradient (i.e., the exponential growth rate) of mode 6 is greater than those of modes 5 and 7 , which are greater than that of mode 8 , while mode 4 grows still more slowly. This agrees exactly with the linear analysis growth rates [see Fig. $5(f)]$ and leads to the selection of the mode 6 pattern.

In Fig. 5, the calculation of the gradient from the numerical data is not very precise, but the estimates presented show good agreement with the linear growth rates of the modes corresponding to the final pattern. It is apparent also that (in these particular numerical simulations) the growth is never faster than it is in this linear phase and hence that the linear growth rates bound the rate of pattern formation.

These results suggest that, typically, our linear analysis results will hold. We find, however, that if the parameters are

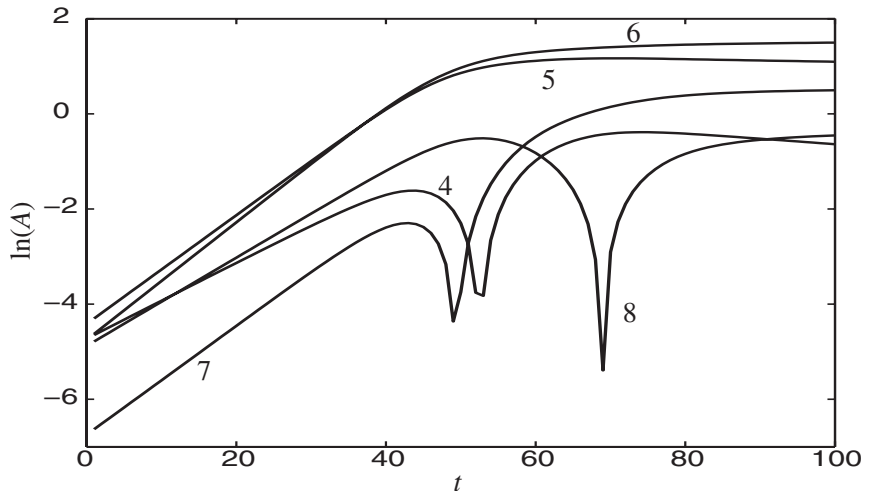

FIG. 6. Time dependence of the natural logarithm of the amplitudes $A$ of modes $4-8$ in the simulation of Fig. 5(c). This plot shows how a mode 6 pattern is selected.

such that the system is very far from the Turing bifurcation, nonlinear interactions can lead to two effects which may speed up the formation of few-peaked patterns.

\section{PEAK COMPETITION}

In the highly nonlinear regime far from bifurcation (for example, when the ratio of diffusion coefficients is very small), peaks, once formed, can compete with one another and some may be annihilated. The modes that initially grow from the uniform steady state are those with large linear growth rates. As we have seen, the growth rates of the fastest modes are not limited by the diffusion rates, provided the ratio of diffusion coefficients is very small, and so the growth of these modes may be very fast. The pattern, having attained sufficient amplitude, may then reorganize such that it consists of only one or a few peaks. Providing that this reorganization is also fast, this process could lead to fewpeaked patterns emerging in reaction diffusion systems in a shorter time than linear analysis suggests is possible. (We assume that cells in the biological system only respond to high levels of activator concentration when those levels are sustained, so that the many transiently appearing peaks have no long term effect.)

Figure 7 shows an example of this phenomenon in a simulation of the Gierer-Meinhardt model. Figure 7(a) shows an early time plot of the activator concentration displaying many peaks, while Fig. 7(b) shows the long time activator profile containing only two peaks (the system takes a very long time to reach steady state, but the number of peaks is stable by this stage and their positions change only slightly on a very long time scale). Figure 7(c) shows the natural logarithm of the amplitude of the pattern and Fig. 7(d) shows the growth rates of modes predicted by linear analysis. This example shows that linear analysis does not always predict the final pattern; however it is not clear from Fig. 7(c) that the initial growth rate is much faster than the linear analysis prediction for mode 4 (the two-peaked mode). Figure 7(c) does, however, reveal another way in which the system may exceed the growth rate predicted by linear analysis and we investigate this in the following section. 


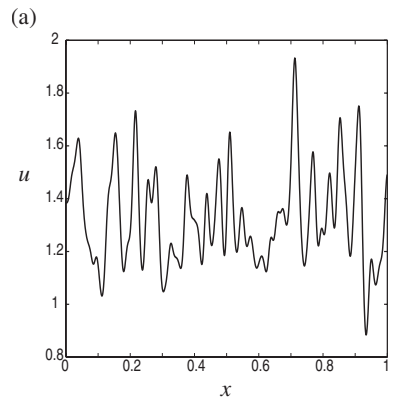

(b)

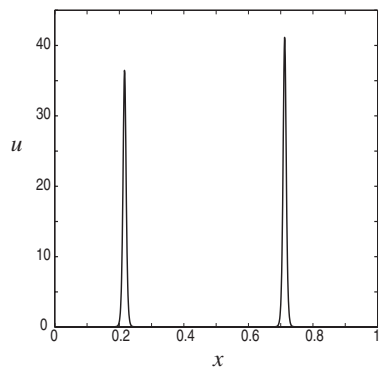

(c)

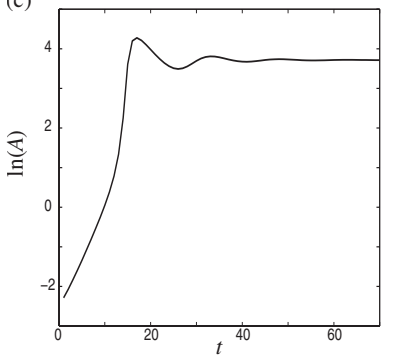

(d)

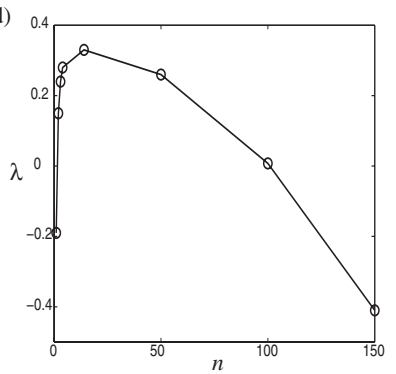

(a)

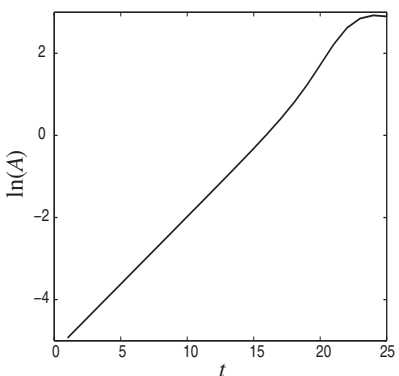

(b)

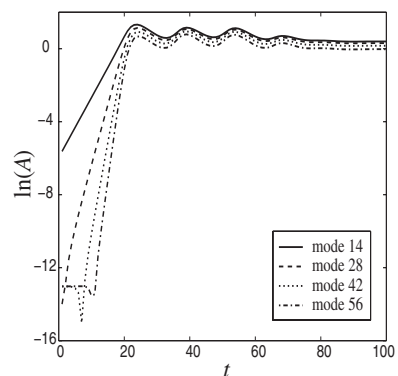

FIG. 7. Peak competition in the Gierer-Meinhardt model with parameter values $D_{v}=0.027, d=0.000125, r=0.006, \nu=0.45$, and $\mu=0.35$. (a) Activator profile $u(x, t)$ at time $t=10$. (b) Activator profile $u(x, t)$ at time $t=100$. (c) Growth profile of the natural logarithm of amplitude $A$ of activator pattern vs time. (d) Growth rates $\lambda$ of modes (with mode number $n$ ) predicted by linear analysis. Mode 14 is predicted to have the maximum growth rate. Simulations in (a)-(c) performed with initial conditions between $\pm 5 \%$ noise, 1001 spatial discretization points, and tolerance $=10^{-9}$.

\section{ACCELERATED GROWTH}

There is a regime of growth [between about $t=12$ and $t$ $=16$ in Fig. 7(c)], where the amplitude of the pattern grows at a faster rate than it does in the linear regime. Indeed, the rate reaches at least 1.3 which is several times faster than the linear growth rate of the fastest growing mode $(0.33$ for mode 14). The average growth rate from $t=1$ to $t=16$ is 0.43 , which again is faster than the linear growth rate of the fastest growing mode. At $t=16$, one of the peaks in the activator concentration is well-established and the other, although still growing, is clearly discernible. This demonstrates that it is possible to establish a pattern in a shorter time than suggested by linear analysis. The accelerated regime of growth is clearly a nonlinear phenomenon. We now investigate why it occurs.

Individual modes only interact with one another via the nonlinear terms. We look at the growth profiles of their amplitudes to see how they contribute to the accelerated growth. In the example of Fig. 7, many modes have positive growth rates and the white noise in the initial conditions means that each of these modes is present in the system. Hence the nonlinear interactions between modes are very complex and many modes contribute to the accelerated growth. We therefore look at a simpler case, when the initial perturbation about the homogeneous steady state is of a single wave number. We choose the wave number with maximal linear growth rate, that is a perturbation of the form $\cos (14 \pi x)$. We choose its amplitude to be $0.2 \%$ of the steady state value, which

FIG. 8. Growth profiles of the natural logarithm of the amplitude $A$ of activator pattern. Parameter values are as in Fig. 7, but initial conditions are a perturbation about the steady state of single wave number, $14 \pi$. (a) Growth of the initial mode for $0 \leqslant t \leqslant 25$ showing the initial linear and the accelerated regimes. (b) Growth of the initial mode and its first three harmonics, during whole simulation time $(0 \leqslant t \leqslant 100)$. Number of spatial discretization points $=1001$, tolerance $=10^{-9}$.

represents roughly the amount likely to be present in approximately white noise with expected magnitude of $5 \%$ of the steady state value on 1000 cells or with expected magnitude $1.4 \%$ on 100 cells.

Figure 8 shows the results of a simulation using these initial conditions. As before, we observe accelerated growth (although it is not as marked as in the previous simulations) followed by peak competition. Figure 8(a) shows the regime of accelerated growth. The average growth rate between $t$ $=19$ and $t=20$ is 0.49 and overall between $t=1$ and $t=20$ it is 0.36 . Both of these values are higher than the maximum linear growth rate $(0.33$ - the growth rate of the mode present in the initial conditions). The acceleration becomes evident when the pattern amplitude is about 0.05 , which is $4 \%$ of the steady state value. During the period of accelerated growth, simulations show that the only modes present (at any significant level) are the initial mode $[\cos (14 \pi x)]$ and its harmonics $[\cos (28 \pi x), \cos (42 \pi x)$, etc. $]$ The pattern during this period consists of seven growing peaks. Later, however, the peaks compete, as in the previous simulations, resulting in only 1.5 peaks). Thus the acceleration must be due to nonlinear harmonic excitations. The harmonics appear in order, the lowest wave number first-so that to start with only the initial mode is present, followed by its first harmonic and so on.

Figure 8(b) shows the growth profile of the initial mode and its first three harmonics. Between $t=18$ and $t=19$, the gradient (i.e., growth rate) of mode 14 is 0.34 , of mode 28 is 0.62 , of mode 42 is 0.90 , and of mode 56 is 1.18 . Thus the growth rate of the mode is roughly proportional to its wave number. This result is in concordance with a set of hypotheses for accelerated growth presented in Appendix D.

\section{CORRELATION BETWEEN SPEED OF DEVELOPMENT AND NUMBER OF PEAKS}

We have seen that although linear analysis can give a good indication in many cases of the maximum rate of growth of a pattern, it is not limiting in all cases. It remains 

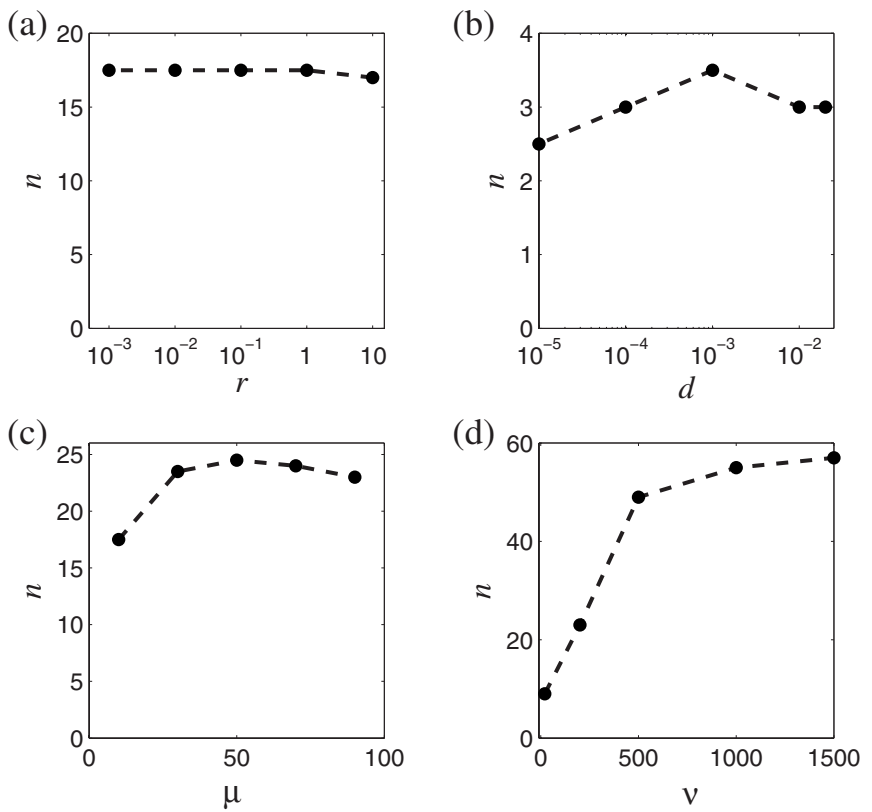

FIG. 9. Dependence of the number of peaks $n$ in solutions of the Gierer-Meinhardt model on each of the model parameters (with all other parameters being held constant). (a) $d=0.01, \mu=10, \nu=100$. (b) $r=0.01, \mu=0.333, \nu=2$. (c) $r=0.01, d=0.01, \nu=100$. (d) $r$ $=0.001, d=0.001, \mu=10$. Note that $r$ and $d$ are plotted on a logarithmic scale in (a) and (b), respectively.

to be demonstrated, however, whether a few-peaked pattern can be established on a sufficiently short time scale for primitive streak initiation, for example, in our model system. In this section, we summarize the results of numerical simulations, using a wide range of parameter values, focusing on the number of peaks in the final pattern and the time taken for the pattern to be established. As we have noted already, care must be taken in using numerical simulation results to infer exact timings of pattern formation. However, our aim here is to infer rough timings and compare the time taken in different regions of parameter space.

Figure 9 shows example simulation results, in which the numbers of peaks in the patterns at time $t=500$ are plotted against the values of a parameter of the system, the other parameters being held constant. The ranges of the parameter being varied are designed to give as broad a view of the Turing space as possible. The results shown are chosen, as far as possible, to be representative. We conclude from these simulation results that the number of peaks increases with $\nu$. The dependence on the other parameters is much weaker. Changing $r$ has negligible effect on the number of peaks. The dependence on $\mu$ is unclear, but it does not seem very marked. Values of $d$ near to bifurcation (i.e., larger values) tend to lead to slightly fewer peaks, but the effect is marginal. For a more detailed analysis of the effects on the pattern of the system parameters see [14].

Thus if we are interested in few-peaked patterns, we should pick a small value of $\nu$ (i.e., a low inhibitor degradation rate, although this should be higher than the activator diffusion rate or the system will start to oscillate) and choose the other parameters so that we are in the Turing space, perhaps taking care that $d$ is not too small. We now assess the
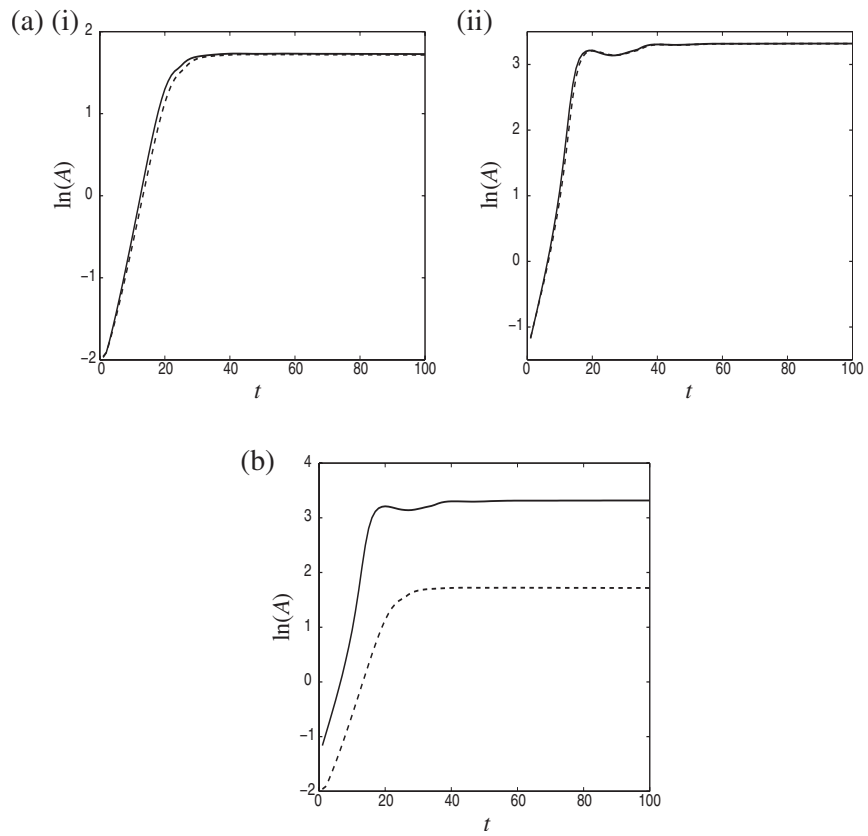

FIG. 10. Dependence of the growth profiles of the natural logarithm of the amplitude $A$ on the parameters $r$ and $d$. In all plots, $\nu=0.4$ and $\mu=0.3$. (a) Growth profiles for $r=0.0001$ (solid line) and $r=0.01$ (dashed line) for (i) $d=0.01$, and (ii) $d=0.0001$. (b) Growth profiles for $r=0.01$ with $d=0.0001$ (solid line) and $d=0.01$ (dashed line). In all simulations, the number of spatial discretization points $=101$, the noise in initial conditions is between $\pm 10 \%$, and the tolerance $=10^{-9}$.

implications of this choice for the growth rates of the patterns.

We find from the results of simulations that we need a value of $\nu$ less than about 0.5 to obtain a one-peaked pattern, which, by the linear analysis in Sec. II, shows that the maximum linear growth rate is less than 0.5 . When $d$ is not too small there will be no regime of accelerated growth and so this restriction on the linear growth rate will mean that the pattern takes at least a time $\approx \frac{1}{0.5}\left[\frac{1}{2} \ln \left(\frac{m}{2}\right)\right] \approx 3.9$ (over ten hours) to be established (see linear analysis section-we assume the threshold level $y$ is at least as high as the noise level $x$ and that we have around 100 cells in our domain). However, if we make $d$ very small (for example $10^{-4}$, which is unlikely from a biological point of view), we can obtain a regime of accelerated growth. In Fig. 10, we use parameter values $\nu=0.4$ and $\mu=0.3$ (about as large as possible to give rise to a one-peaked pattern). The figure shows that $r$ has very little effect on the growth profile, but that reducing $d$ can lead to accelerated growth. The pattern takes a time $t$ $\approx 3.6$ (which corresponds to $10 \mathrm{~h}$ ) to reach $20 \%$ amplitude when $d=10^{-4}$, with initial conditions uniformly distributed between $\pm 10 \%$ noise on a domain of 100 cells, and a time $t \approx 9.9$ (which corresponds to over $27 \mathrm{~h}$ ) when $d=0.01$. Even when the pattern reaches the requisite amplitude it has not always yet become single-peaked.

We have performed similar numerical simulations with Schnakenberg or depletion and glycolysis kinetics and both appear to be too slow to explain, for example, primitive streak initiation. Our simulations suggest that a two-species 


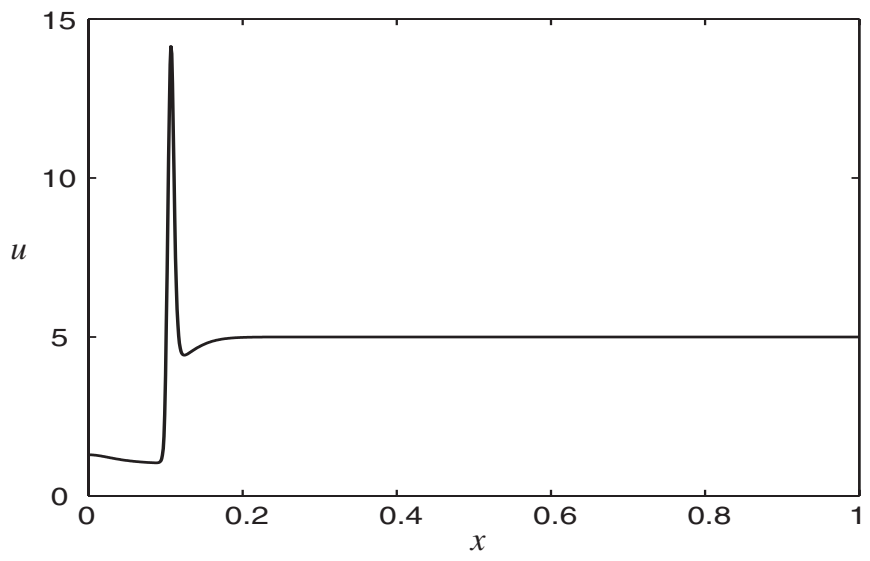

FIG. 11. A typical one-peaked pattern in the activator concentration $u$ resulting from spatial inhomogeneity in the parameter $r$. In this example, the spatially uniform parameters are $\nu=20, \mu=10$, $d=0.001$. The value of $r$ changes abruptly at $x=0.1$, from $r=10$ for $x<0.1$ to $r=30$ for $x>0.1$. Only for $x<0.1$ does the system lie within the Turing space. The uniform steady state value of $u$ corresponding to the parameters on $x<0.1$ is $u_{0}=3$ and the value corresponding to the parameters on $x>0.1$ is $u_{0}=5$. Number of spatial discretization points $=1001$, tolerance $=10^{-9}$.

reaction diffusion system with an inhibitor diffusion coefficient of less than $10^{-6} \mathrm{~cm}^{2} / \mathrm{s}$ would take several (>2) days to initiate a single-peaked pattern on a domain of length $6 \mathrm{~mm}$ (or equivalently a gradient on a domain of length $3 \mathrm{~mm}$ ) from initial conditions of $\pm 10 \%$ noise about the uniform steady state. Many-peaked patterns can be initiated more rapidly.

\section{EFFECT OF SPATIALLY VARYING PARAMETERS ON PATTERNING SPEED}

In the previous section, we established a link between the speed of development of a pattern and its wavelength. Now, by varying the parameters of a reaction diffusion system, we can move in and out of the Turing space. Thus if the parameters are allowed to vary in space, then in some sections of the domain the parameters of the system may be within the Turing space and in others they may not. This can lead to sections of the domain becoming patterned, while others remain unpatterned $[15,14]$. Specifically, if the parameters are such that on a small section of the domain a short wavelength pattern develops rapidly, but on the remainder of the domain no pattern forms, then this may give rise to a singlepeaked or few-peaked pattern in a short time.

Figure 11 shows an example of a simulation performed on an inhomogeneous domain of this type. Since there are two distinct homogeneous steady state values, we cannot say when the pattern amplitude has reached $y \%$ of the steady state value. However, it is well established before $t=1$, that is, before three hours have elapsed. We performed simulations with various initial conditions, setting the activator and inhibitor equal to the uniform steady state values on the left hand side, to the value on the right hand side and to the step-function state. Varying amounts of noise were added to these initial conditions. We found that by $t=1$, the solution (still growing) was almost independent of the initial conditions. Thus with a spatially varying parameter, a one-peaked pattern can be initiated in $3 \mathrm{~h}$ in a way that is not sensitively dependent on the details of the noise in the system (and hence on the number of cells).

The use of a spatially varying parameter, however, pushes back the problem to explaining the patterning of that parameter. It is clear, though, that in embryonic development the patterning is sequential and all but the first patterning events take place in a domain which already has some structure (i.e., a "prepattern"). The importance of these hierarchies of pattern was noted by Turing [11]. Our simulations demonstrate that secondary patterning in a reaction diffusion system can be considerably faster than patterning de novo. It is also clear that parameter values can be influenced by external physical factors in a much more obvious way than can the concentrations of gene products directly. For example, before the initiation of the primitive streak in the chick embryo appears to have started, there is already a bias in the embryo. The cell density in the posterior is greater than that in the anterior and this is thought to be due to the effects of gravity when the egg is tilted (rendering the presumptive posterior of the embryo uppermost) in the oviduct (see [16]). If we were to suppose that the activator was produced at a given rate per cell then this would naturally lead to a preexisting gradient in an activator production parameter.

In Fig. 11, the activator pattern is much sharper than the original parameter inhomogeneity. This shows that it is possible for secondary patterns to refine and sharpen the existing structure of the biological system. This secondary pattern does not, however, lend itself to regulation, which is an essential feature of many patterning processes during embryonic development. If much of the domain has parameters outside the Turing space, then, in isolation, those sections will not regenerate pattern unless the underlying pattern in the parameter itself regulates.

We note that an alternative to having prepatterned parameters in the reaction diffusion system is to have boundary conditions that somehow force the patterning. Thus, for example, boundary conditions that are inconsistent with the homogeneous steady state can also speed up patterning.

\section{CONCLUSIONS}

According to linear analysis in a two-component Turing system, the time taken for the establishment of a pattern with $k$ half peaks from initial state of a small perturbation about the uniform steady state is at least $A L^{2} /\left(D_{v} k^{2}\right)$, where $A$ is a constant depending on the kinetic parameters of the system, the details of the initial conditions and the amplitude that the pattern has to reach. So, according to linear analysis, the time taken to establish a pattern has a very similar form to that for gradient formation by simple diffusion [2]. This also shows that the time taken depends on the wavelength of the pattern-long wavelength patterns are slow to develop.

The details of the noise in the initial conditions are important in determining the time taken for a pattern to develop. If the noise is added pointwise at each cell or discretization point of the system, then as the number of cells or 
discretization points increases, the Fourier components of the noise become vanishingly small and hence the time taken for a pattern to form becomes unboundedly large.

Typically, patterns do grow initially at roughly the rate predicted by linear analysis and then the growth slows down and stops. The bounds obtained from linear analysis will therefore usually bound above the rate of patterning of the full nonlinear system.

Far from bifurcation two phenomena occur which mean that the linear bounds do not apply: (i) peak competition-a rapidly growing short wavelength pattern may appear initially, but then the peaks compete and some are annihilated; (ii) accelerated growth-nonlinear mode interactions can cause a regime of growth which is faster than the initial growth in the linear regime.

Despite the fact that bounds from linear theory do not always apply, simulations show that if the diffusion coefficients are small, then the patterns can be fast-growing only if they have short wavelength. It seems that straightforward reaction diffusion systems cannot explain fast long wavelength patterning in biological systems, such as is required in the initiation of the primitive streak.

If the parameters of the reaction diffusion system have a dependence on space, then a single peak or few-peaked pattern can be established rapidly (see $[13,15,14]$ ). Nature employs a variety of strategies to ensure that patterning typically takes place on a domain which already has some spatial structure. Embryonic development involves a hierarchy of patterning and reaction diffusion may be able to explain rapid secondary patterning of a long wavelength, although it cannot explain rapid de novo patterning.

In this paper, we have focused on a few aspects of the patterning process which affect the speed of formation of patterns. We chose to focus on a few well-studied kinetic schemes. Future research might consider how to generalize the ideas we have proposed to other kinetic schemes and to consider other aspects of the patterning process and how they affect the speed of patterning.

\section{ACKNOWLEDGMENTS}

K.M.P. acknowledges the Joint Research Councils (EPSRC, BBSRC, and MRC) for a Lectureship in Bioinformatics. N.A.M.M. acknowledges the support of BBSRC (Grant No. 50/MMI09729), the Isaac Newton Institute for Mathematical Sciences, University of Cambridge, for financial assistance by EPSRC, and the University of Sheffield for financial assistance. P.K.M. acknowledges financial support from the Royal Society Leverhulme Trust and thanks the National Centre for Theoretical Sciences, Taiwan and Hokkaido University, Sapporo, for partial support.

\section{APPENDIX A: NONDIMENSIONALIZATION OF THE GIERER-MEINHARDT MODEL}

The Gierer-Meinhardt model equations are given by

$$
\left.\begin{array}{l}
u_{t}=k_{1}+k_{3} \frac{u^{2}}{v}-k_{2} u+D_{u} u_{x x} \\
v_{t}=k_{4} u^{2}-k_{5} v+D_{v} v_{x x}
\end{array}\right\} \text { on }[0, L],
$$

where $u$ is the activator concentration, $v$ is the inhibitor concentration, and $k_{1}, \ldots, k_{5}$ are positive parameters, see [7]. To be fully prescribed the equations also require boundary conditions on $u$ and $v$ at $x=0, L$.

We introduce nondimensional quantities

$$
\begin{gathered}
\bar{x}=\frac{x}{L}, \quad \bar{t}=\frac{t}{T}, \quad \bar{u}=\frac{u k_{4}}{k_{3}}, \quad \bar{v}=\frac{v k_{4}^{2} k_{1}}{k_{3}^{3}}, \\
r=\frac{k_{1} k_{4} T}{k_{3}}, \quad \mu=T k_{2}, \quad \nu=T k_{5}, \quad \bar{D}_{u}=\frac{T D_{u}}{L^{2}}, \quad \bar{D}_{v}=\frac{T D_{v}}{L^{2}},
\end{gathered}
$$

where $T$ is a fixed time scale-here we take $T=10^{4} \mathrm{~s}$.

On substituting into Eq. (A1) these yield

$$
\left.\begin{array}{l}
\bar{u}_{\bar{t}}=r\left(\frac{\bar{u}^{2}}{\bar{v}}+1\right)-\mu \bar{u}+\bar{D}_{u} \bar{u}_{\overline{x x}} \\
\bar{v}_{\bar{t}}=r \bar{u}^{2}-\nu \bar{v}+\bar{D}_{v} \bar{v}_{\overline{x x}}
\end{array}\right\} \text { on }[0,1] .
$$

\section{APPENDIX B: LINEAR ANALYSIS BOUNDS}

The linear growth rates of the cosine mode perturbations, $\cos (k x)$, about the homogeneous steady state solution of a reaction diffusion system are given by the eigenvalues of the matrix $J-D k^{2}$ given by

$$
J-D k^{2}=\left(\begin{array}{cc}
f_{u}-D_{u} k^{2} & f_{v} \\
g_{u} & g_{v}-D_{v} k^{2}
\end{array}\right) .
$$

If we let the trace of this matrix be denoted by $\operatorname{tr}\left(k^{2}\right)$ and the determinant by $\Delta\left(k^{2}\right)$, then the growth rates $\lambda_{+}\left(k^{2}\right)$ and $\lambda_{-}\left(k^{2}\right)$ are the roots of the equation

$$
\lambda^{2}-\operatorname{tr}\left(k^{2}\right) \lambda+\Delta\left(k^{2}\right)=0 .
$$

We are interested only in the root with largest real part $\left(\lambda_{+}\right)$. We differentiate Eq. (B2) with respect to $k^{2}$ and rearrange to give

$$
\frac{\partial \lambda_{+}}{\partial k^{2}}=\frac{\lambda_{+}\left(\partial \mathrm{tr} / \partial k^{2}\right)-\partial \Delta / \partial k^{2}}{\sqrt{\operatorname{tr}^{2}-4 \Delta}} .
$$

Setting $\frac{\partial \lambda_{+}}{\partial k^{2}}=0$, substituting in the expression for $\lambda_{+}$[solution to the quadratic Eq. (B2) with $+\sqrt{ }$, and rearranging yields a quadratic in $k^{2}$. If this has a positive solution (it can have only one, as discussed below) then its positive square root, $k=k_{*}$, is the value of $k$ for which $\lambda_{+}$takes its maximal value, $\left(\lambda_{+}\right)_{\max }$. This value of $k$ is given by

$$
k_{*}^{2}=\frac{g_{v}-f_{u}+\left(D_{u}+D_{v}\right) \sqrt{-f_{u} g_{v} / D_{u} D_{v}}}{D_{v}-D_{u}} .
$$

The other root (with $-\sqrt{\frac{-f_{u} g_{v}}{D_{u} D_{v}}}$ instead of + ), is negative (and hence $k$ imaginary), since $D_{v}>D_{u}, f_{u}>0$, and $g_{v}<0$ in the Turing space. Substituting this into the expression for $\lambda_{+}$and rearranging yields 


$$
\begin{gathered}
\left(\lambda_{+}\right)_{\max }=f_{u}+\frac{D_{u}\left(f_{u}-g_{v}\right)-2 \sqrt{-f_{v} g_{u} D_{u} D_{v}}}{D_{v}-D_{u}} \\
=f_{u}+\frac{\left(g_{v}-f_{u}\right) D_{u}-2 D_{u} D_{v} k_{*}^{2}}{D_{u}+D_{v}} .
\end{gathered}
$$

Equation (B5) is the expression given in Eq. (11) and from Eq. (B6) it is clear that $\left(\lambda_{+}\right)_{\max } \leqslant f_{u}$ [if $\left(\lambda_{+}\right)_{\max }$ exists then $k_{*}^{2}>0$, and also to be in Turing space requires $g_{v}<0$ and $\left.f_{u}>0\right]$. Thus

$$
\left(\lambda_{+}\right)_{\max }\left(D_{u}, D_{v}, J\right) \leqslant\left(\lambda_{+}\right)_{\max }\left(0, D_{v}, J\right)=f_{u} .
$$

We now consider bounds on the value of $\lambda_{+}\left(k^{2}\right)$ for a fixed mode $k$ as the parameters of the system are varied. Let $\alpha$ be a general parameter, then differentiating Eq. (B2) and rearranging, we get

$$
\frac{\partial \lambda_{+}}{\partial \alpha}=\frac{\lambda_{+}(\partial \mathrm{tr} / \partial \alpha)-\partial \Delta / \partial \alpha}{\sqrt{\operatorname{tr}^{2}-4 \Delta}} .
$$

In the case of the Gierer-Meinhardt model, we have

$$
\begin{gathered}
\operatorname{tr}\left(k^{2}\right)=-\nu-\mu+\mu \frac{2 \nu}{\nu+r}-\left(D_{u}+D_{v}\right) k^{2}, \\
\Delta\left(k^{2}\right)=\mu \nu+\left(\nu D_{u}+\mu D_{v}-\mu D_{v} \frac{2 \nu}{\nu+r}\right) k^{2}+D_{u} D_{v} k^{4} .
\end{gathered}
$$

Differentiating with respect to $r$, we find

$$
\begin{aligned}
& \frac{\partial \mathrm{tr}}{\partial r}=-\mu \frac{2 \nu}{(\nu+r)^{2}}<0, \\
& \frac{\partial \Delta}{\partial r}=\mu D_{v} \frac{2 \nu}{(\nu+r)^{2}}>0,
\end{aligned}
$$

so if $\lambda_{+}>0\left(\Rightarrow \sqrt{\operatorname{tr}^{2}-4 \Delta}\right.$ is real and positive $)$, then $\frac{\partial \lambda_{+}}{\partial r}<0$. So if we fix the other parameters, then we must have

$$
\lambda_{+}\left(r, D_{u}, D_{v}, \nu, \mu ; k^{2}\right) \leqslant \max \left\{0, \lambda_{+}\left(r_{0}, D_{u}, D_{v}, \nu, \mu ; k^{2}\right)\right\},
$$

where $r_{0}$ is the minimum value of $r$, subject to the constraint that the uniform steady state is stable to homogeneous perturbations. Analysis of the ordinary differential equation system shows that

$$
r_{0}=\left\{\begin{array}{ll}
\nu \frac{\mu-\nu}{\nu+\mu} & \mu \geqslant \nu \\
0 & \mu \leqslant \nu
\end{array} .\right.
$$

We first assume that $\mu \geqslant \nu$, substituting the expression for $r_{0}$ into those for tr, $\Delta$ and hence for $\lambda_{+}$and rearranging, we get

$\left(\lambda_{+}\right)_{0}$

$$
\begin{aligned}
& \equiv \lambda_{+}\left(r_{0}, D_{u}, D_{v}, \nu, \mu\right) \\
& =\frac{-k^{2}\left(D_{u}+D_{v}\right)+\sqrt{k^{4}\left(D_{v}-D_{u}\right)^{2}+4 \nu\left(D_{v}-D_{u}\right) k^{2}-4 \nu \mu}}{2} .
\end{aligned}
$$

Setting $z=k^{2}\left(D_{v}-D_{u}\right)$ gives

$$
\begin{aligned}
\left(\lambda_{+}\right)_{0} & =\frac{-z-2 D_{u} k^{2}+\sqrt{z^{2}+4 \nu z-4 \nu \mu}}{2} \\
& <\frac{-z+\sqrt{z^{2}+4 \nu z-4 \nu \mu}}{2} .
\end{aligned}
$$

Now $\left(\frac{z}{2 \mu}-1\right)^{2}>0 \Rightarrow \frac{z^{2}}{4 \mu}>z-\mu$, which implies

$$
\left(\lambda_{+}\right)_{0}<\frac{-z+\sqrt{z^{2}+(\nu / \mu) z^{2}}}{2}=\frac{z(\sqrt{(\nu / \mu)+1}-1)}{2} .
$$

We have assumed here that $\nu \leqslant \mu$, so

$$
\left(\lambda_{+}\right)_{0}<\frac{(\sqrt{2}-1)}{2} k^{2}\left(D_{v}-D_{u}\right)<\frac{(\sqrt{2}-1)}{2} D_{v} k^{2} .
$$

We now assume that $\mu \leqslant \nu$ and hence $r_{0}=0$. This means that

$$
\operatorname{tr}_{0} \equiv \operatorname{tr}\left(k^{2}\right)\left(r=r_{0}\right)=\mu-\nu-\left(D_{u}+D_{v}\right) k^{2},
$$

$$
\Delta_{0} \equiv \Delta\left(k^{2}\right)\left(r=r_{0}\right)=\mu \nu+\left(\nu D_{u}-\mu D_{u}\right) k^{2}+D_{u} D_{v} k^{4},
$$

and hence $\frac{\partial \mathrm{rt}_{0}}{\partial \nu}=-1<0$ and $\frac{\partial \Delta_{0}}{\partial \nu}=\mu+D_{u} k^{2}>0$. So if $\left(\lambda_{+}\right)_{0}$ $>0, \frac{\partial\left(\lambda_{+}\right)_{0}}{\partial \nu}<0$. Thus $\left(\lambda_{+}\right)_{0} \leqslant \max \left\{0,\left(\lambda_{+}\right)_{0}(\nu=\mu)\right\}$. We have already bounded $\left(\lambda_{+}\right)_{0}(\nu=\mu)$ in Eq. (B17) and the bound is $>0$. So

$$
\left(\lambda_{+}\right)_{0}<\frac{(\sqrt{2}-1)}{2} D_{v} k^{2},
$$

$\forall r, D_{u}, D_{v}, \mu, \nu$ in the Turing space. This is the bound given in Eq. (13).

The above bound is specific to the Gierer-Meinhardt model. We look now for a bound on $\lambda_{+}\left(k^{2}\right)$ valid for a general two-species reaction-diffusion system. We have shown that in a Turing system there can exist only one positive real value $k=k_{*}$ for which $\frac{\partial\left(\lambda_{+}\right)_{0}}{\partial k^{2}}=0$. Differentiating Eq. (B2) twice and simplifying yields

$$
\left(2 \lambda_{+}-\operatorname{tr}\right) \frac{\partial^{2}\left(\lambda_{+}\right)_{0}}{\partial\left(k^{2}\right)^{2}}=2 \frac{\partial\left(\lambda_{+}\right)_{0}}{\partial k^{2}} \frac{\partial \operatorname{tr}}{\partial k^{2}}-2\left(\frac{\partial\left(\lambda_{+}\right)_{0}}{\partial k^{2}}\right)^{2}-\frac{\partial^{2} \Delta}{\partial\left(k^{2}\right)^{2}}
$$


and $\frac{\partial \mathrm{tr}}{\partial k^{2}}=-\left(D_{u}+D_{v}\right)<0$ and $\frac{\partial^{2} \Delta}{\partial\left(k^{2}\right)^{2}}=2 D_{u} D_{v}>0$. Now $\operatorname{tr}\left(k^{2}\right)$ $<0 \forall k^{2}$, so if $\lambda_{+}>0$ then $2 \lambda_{+}-\operatorname{tr}>0$. So $\forall k^{2}$ with $\lambda_{+}>0$, $\frac{\partial^{2}\left(\lambda_{+}\right)_{0}}{\partial\left(k^{2}\right)^{2}}<0$ if $\frac{\partial\left(\lambda_{+}\right)_{0}}{\partial k^{2}}>0$. This means that

$$
\frac{\partial\left(\lambda_{+}\right)_{0}}{\partial k^{2}}<\frac{\partial\left(\lambda_{+}\right)_{0}}{\partial k^{2}}\left(k^{2}=\bar{k}^{2}\right),
$$

$\forall k^{2}$ with $\lambda_{+}\left(k^{2}\right)>0$, where $\bar{k}^{2}$ is the smaller root of $\lambda_{+}=0$. Thus

$$
\begin{aligned}
\lambda_{+}\left(k^{2}\right)< & \max \left\{0,\left(k^{2}-\bar{k}^{2}\right) \frac{\partial\left(\lambda_{+}\right)_{0}}{\partial k^{2}}\left(k^{2}=\bar{k}^{2}\right)\right\} \\
& <k^{2} \frac{\partial\left(\lambda_{+}\right)_{0}}{\partial k^{2}}\left(k^{2}=\bar{k}^{2}\right) .
\end{aligned}
$$

Now $\frac{\partial \mathrm{tr}}{\partial D_{u}}<0$ and $\frac{\partial \Delta}{\partial D_{u}}>0$, so

$$
\begin{gathered}
\lambda_{+}\left(k^{2} ; D_{u}, D_{v}, J\right) \leqslant \max \left\{0, \lim _{D_{u} \rightarrow 0}\left[\lambda_{+}\left(k^{2} ; D_{u}, D_{v}, J\right)\right]\right\} \\
<k^{2} \lim _{D_{u} \rightarrow 0}\left[\frac{\partial \lambda_{+}}{\partial k^{2}}\left(k^{2}=\bar{k}^{2} ; D_{u}, D_{v}, J\right)\right] .
\end{gathered}
$$

Setting $D_{u}=\epsilon$, where $\epsilon$ is small, we find that the equation $\lambda_{+}\left(k^{2}\right)=0\left[\Leftrightarrow \Delta\left(k^{2}\right)=0\right]$ has a solution which is $O(1)$ and solution which is $O\left(\frac{1}{\epsilon}\right)$. Since $\bar{k}^{2}$ is the smaller root it is $O(1)$. To leading order it is given by

$$
\bar{k}^{2}=\frac{f_{u} g_{v}-f_{v} g_{u}}{f_{u} D_{v}}
$$

and substituting this into the equation for $\frac{\partial \lambda_{+}}{\partial k^{2}}$, we get

$$
\frac{\partial \lambda_{+}}{\partial k^{2}}\left(k^{2}=\bar{k}^{2}\right)=\frac{f_{u} D_{v}}{\beta-1}
$$

to leading order and hence in the limit as $D_{u} \rightarrow 0$, where $\beta$ $=\frac{\left|f_{v} g_{u}\right|}{f_{u}^{2}}$. Thus from Eq. (B25),

$$
\lambda_{+}\left(k^{2}\right)<\frac{D_{v} k^{2}}{\beta-1} .
$$

This is the bound given in Eq. (14).

\section{APPENDIX C: EFFECT OF INITIAL CONDITIONS}

In our discrete system, we define discrete analogs of the Fourier components:

$$
A_{n}=\frac{2}{p} \sum_{r=1}^{p} u(r) \cos \left(\frac{n \pi r}{p}\right), \quad n=1,2, \ldots
$$

[cf. $A_{n}=2 \int_{0}^{1} u(x) \cos (n \pi x) d x$ - the discrete analog will give a good approximation to the Fourier components of a continuous function if $p \gg n$; the discrepancy will be $O(1 / p)]$. Substituting for $u$, the constant component contributes only a term of order $1 / p$ to the nonconstant Fourier modes. So

$$
A_{n}=\frac{2 u_{0}}{p} \sum_{r=1}^{p} \xi(r) \cos \left(\frac{n \pi r}{p}\right)+O\left(\frac{1}{p}\right) .
$$
$A_{n}$ :

We calculate the expected value and the variance for each

$$
\begin{aligned}
E\left(A_{n}\right) & =E\left[\frac{2 u_{0}}{p} \sum_{r=1}^{p} \xi(r) \cos \left(\frac{n \pi r}{p}\right)\right]+O\left(\frac{1}{p}\right) \\
& =\frac{2 u_{0}}{p} \sum_{r=1}^{p} E[\xi(r)] \cos \left(\frac{n \pi r}{p}\right)+O\left(\frac{1}{p}\right) .
\end{aligned}
$$

Now $E[\xi(r)]=0 \forall r$, so

$$
E\left(A_{n}\right)=0+O\left(\frac{1}{p}\right),
$$

the second term being a correction due to the discretization of the integral,

$$
\operatorname{Var}\left(A_{n}\right) \equiv E\left(A_{n}^{2}\right)-\left[E\left(A_{n}\right)\right]^{2}=E\left(A_{n}^{2}\right)-O\left(\frac{1}{p^{2}}\right),
$$

and

$$
\begin{aligned}
E\left(A_{n}^{2}\right)= & E\left[\frac{4 u_{0}^{2}}{p^{2}} \sum_{r=1}^{p} \xi(r) \cos \left(\frac{n \pi r}{p}\right) \sum_{s=1}^{p} \xi(s) \cos \left(\frac{n \pi s}{p}\right)\right] \\
& +O\left(\frac{1}{p^{2}}\right) \\
= & \frac{4 u_{0}^{2}}{p^{2}} \sum_{r, s=1}^{p} E[\xi(r) \xi(s)] \cos \left(\frac{n \pi r}{p}\right) \cos \left(\frac{n \pi s}{p}\right) \\
& +O\left(\frac{1}{p^{2}}\right)
\end{aligned}
$$

Now $\xi(r)$ and $\xi(s)$ are independent for $r \neq s$ and hence

$$
E[\xi(r) \xi(s)]=E[\xi(r)] E[\xi(s)]=O\left(\frac{1}{p^{2}}\right) .
$$

So

$$
E\left(A_{n}^{2}\right)=O\left(\frac{1}{p^{2}}\right)+\frac{4 u_{0}^{2}}{p^{2}} \sum_{r=1}^{p} E\left[\xi(r)^{2}\right] \cos ^{2}\left(\frac{n \pi r}{p}\right)
$$

and since $E(\xi)=0$,

$$
\begin{aligned}
\operatorname{Var}\left(A_{n}\right)= & O\left(\frac{1}{p^{2}}\right)+\frac{4 u_{0}^{2}}{p^{2}} \operatorname{Var}(\xi) \sum_{r=1}^{p} \cos ^{2}\left(\frac{n \pi r}{p}\right) \\
= & O\left(\frac{1}{p^{2}}\right)+\frac{4 u_{0}^{2}}{p} \operatorname{Var}(\xi)\left[\int_{0}^{1} \cos ^{2}(n \pi x) d x\right. \\
& \left.+O\left(\frac{1}{p}\right)\right] \quad \text { for } p \gg n \\
= & \frac{4 u_{0}^{2}}{p} \operatorname{Var}(\xi) \frac{1}{2}+O\left(\frac{1}{p^{2}}\right)
\end{aligned}
$$

So, to leading order, 


$$
\operatorname{Var}\left(A_{n}\right)=\frac{2 u_{0}^{2}}{p} \operatorname{Var}(\xi)
$$

and

$$
\sigma\left(A_{n}\right)=\sqrt{\frac{2}{p}} u_{0} \sigma(\xi),
$$

where $\sigma$ is the standard deviation.

Now for a random variable $X$,

$$
\operatorname{Var}(|X|) \geqslant 0 \Rightarrow E\left(X^{2}\right) \geqslant[E(|X|)]^{2},
$$

so

$$
E\left(\left|A_{n}\right|\right) \leqslant \sigma\left(A_{n}\right)
$$

and Chebychev's inequality states that

$$
P(|X| \geqslant a) \leqslant \frac{E(|X|)}{a},
$$

so

$$
P\left(\left|A_{n}\right| \geqslant a\right) \leqslant \frac{\sigma\left(A_{n}\right)}{a}=\sqrt{\frac{2}{p}} u_{0} \frac{\sigma(\xi)}{a} .
$$

So, as

$$
p \rightarrow \infty, E\left(\left|A_{n}\right|\right) \rightarrow 0, \quad \text { and } P\left(\left|A_{n}\right| \geqslant a\right) \rightarrow 0, \quad \forall a>0 .
$$

Thus as we increase the number of spatial points in our discretization or cells in our system, the Fourier components become vanishingly small with probability 1 . This means that the time taken to form pattern will increase unboundedly with $p$.

\section{APPENDIX D: MODE-NUMBER DEPENDENCE OF MODE GROWTH RATES}

The dominant term in the rate of change of the initial mode is the linear term. Hence the amplitude of the initial mode $\propto e^{r t}$, where $r$ is its linear growth rate. Since the initial mode has a much larger amplitude than its first harmonic, the dominant term in the rate of change of the first harmonic is due to the squared term in the initial mode rather than the linear term in the first harmonic.

The reaction diffusion equations that we study are of the form

$$
\underline{u}_{t}=\underline{f}(\underline{u})+D \underline{u}_{x x} .
$$

Linearizing about the uniform steady state, $\underline{u}_{0}$, with $\underline{u}=\underline{u}_{0}$ $+\underline{\tilde{u}}$ and $\underline{\tilde{u}}$ small, we get

$$
\underline{\tilde{u}}_{t}=\underline{f}\left(\underline{u}_{0}+\underline{\tilde{u}}\right)-\underline{f}\left(\underline{u_{0}}\right)+D \underline{\tilde{u}}_{x x},
$$

which gives

$$
\mathcal{L} \underline{\underline{u}}=\mathcal{F}_{2}(\underline{\tilde{u}}, \underline{\tilde{u}})+\text { cubic terms }+\cdots,
$$

where $\mathcal{L}$ is the linearized operator about $\underline{u}_{0}$ and $\mathcal{F}_{2}$ is a bilinear quadratic operator. We define the matrix $M_{2}$ by $\mathcal{F}_{2}(\underline{v}, \underline{w}) \equiv \underline{v}^{T} M_{2} \underline{w}, \forall \underline{v}, \underline{w}$.

Letting the amplitude of mode $n$ be denoted by $\underline{A}_{n}(t)$, we have

$$
\underline{\tilde{u}}(t)=\sum_{n} \underline{A}_{n}(t) \cos (n \pi x) \approx \underline{A}_{14}(t) \cos (14 \pi x)
$$

to leading order. Hence

$$
\mathcal{F}_{2}(\underline{\tilde{u}}, \underline{\tilde{u}}) \approx \underline{A}_{14}^{T} M_{2} A_{14} \cos ^{2}(14 \pi x)
$$

$$
=\frac{1}{2} \underline{A}_{14}^{T} M_{2} \underline{A}_{14}[1+\cos (28 \pi x)] \text {. }
$$

$\underline{A}_{28}(t)$ therefore approximately satisfies the following equation:

$$
\begin{aligned}
\left(\frac{\partial}{\partial t}-\left[J-D(28 \pi)^{2}\right]\right) \underline{A}_{28}(t) & =\frac{1}{2} \underline{A}_{14}^{T}(t) M_{2} \underline{A}_{14}(t) \\
& =e^{2 r t} \frac{1}{2} \underline{A}_{14}^{T}(0) M_{2} \underline{A}_{14}(0),
\end{aligned}
$$

where $J$ is the Jacobian matrix. This has solution

$$
\underline{A}_{28}(t)=\underline{a} e^{2 r t}+\underline{b} e^{\lambda_{28} t},
$$

for some constants $\underline{a}$ and $\underline{b}$. Since $r>\lambda_{28}$, we have to leading order

$$
\underline{A}_{28}(t) \propto e^{2 r t} .
$$

For higher harmonics, the nonlinear terms also dominate, and so, using trigonometric identities, we deduce that the main contributions to mode $\cos (14 n \pi x)$ come from products of terms of the form $\Pi_{i=1}^{m} \cos \left(14 r_{i} \pi x\right)$, where the $r_{i}$ are integers such that $\sum_{i=1}^{m} r_{i}=n$. For example, $\frac{d \underline{A}_{42}}{d t} \approx \frac{1}{2} \mathcal{F}_{2}\left(\underline{A}_{14}, \underline{A}_{28}\right)$ $+\frac{1}{4} \mathcal{F}_{3}\left(\underline{A}_{14}, \underline{A}_{14}, \underline{A}_{14}\right)$, where $\mathcal{F}_{3}$ is the cubic term in the expansion of $\underline{f}$. Hence by induction, we get $\underline{A}_{14 n} \propto e^{n r t}$.
[1] T. Miura and P. K. Maini, Bull. Math. Biol. 66, 627 (2004).

[2] F. Crick, Nature (London) 225, 420 (1970).

[3] A. D. Lander, Q. Nie, and F. Y. M. Wan, Dev. Cell 2, 785 (2002).

[4] N. A. M. Monk, Bull. Math. Biol. 60, 901 (1998).

[5] J. Jullien and J. Gurdon, Genes Dev. 19, 2682 (2005).

[6] K. M. Page, P. K. Maini, N. A. M. Monk, and C. D. Stern, J.
Theor. Biol. 208, 419 (2001).

[7] A. Gierer and H. Meinhardt, Kybernetik 12, 30 (1972).

[8] J. Schnakenberg, J. Theor. Biol. 81, 389 (1979).

[9] M. Ashkenazi and H. G. Othmer, J. Math. Biol. 5, 305 (1978).

[10] J. D. Murray, Mathematical Biology, Biomathematics Texts Vol. 19 (Springer-Verlag, Heidelberg, 1989).

[11] A. M. Turing, Phil. Trans. Roy. Soc, series B 237, 37 (1952). 
[12] Y. Chen and A. F. Schier, Curr. Biol. 12, 2124 (2002).

[13] K. M. Page, Ph.D. thesis, University of Oxford, 1999.

[14] K. M. Page, P. K. Maini, and N. A. M. Monk, Physica D 202, 95 (2005).
[15] K. M. Page, P. K. Maini, and N. A. M. Monk, Physica D 181, 80 (2003).

[16] S. Kochav and H. Eyal-Giladi, Science 171, 1027 (1971). 